\title{
A New Analytical Method of Standby System Reliability Model
}

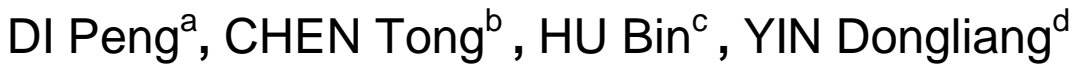

\begin{abstract}
Dept. of Management Engineering and Equipment Economic, Naval Univ. of Engineering, Wuhan 430033, China
\end{abstract}

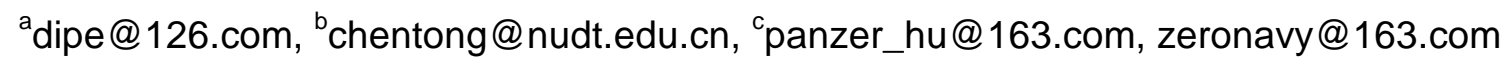

\begin{abstract}
Keywords: general distribution; N-units; warm standby; system reliability
Abstract. Aiming at the problem that the system reliability modeling constraints are too strict in the past studies. A general distribution which approximates an arbitrary distribution is utilized for the $\mathrm{N}$-units warm standby system that has a single maintenance station. The lifetime and repair time of operating and standby units are assumed by the distribution to obtained for a series of reliability parameters, such as system steady-state availability, mean time between failures, ect. Finally, the correctness and applicability of this method are verified by an example analysis.
\end{abstract}

\section{Introduction}

In the design of ship equipment, standby is often a favored guarantee for system reliability ${ }^{[1-3]}$. In a cold standby system, it is assumed that the failure rate of standby unit is zero, so its reliability analysis is normally easier than that of warm standby system ${ }^{[4-6]}$. However, a standby unit of ship equipment may still fail under the humidity and salt mist of marine environment, the high temperature and vibration inside cabin, the shake of ship during high sea condition, and the influence of other environmental factors, even if it is in the cold standby state. Hence, it is more practical to assume that a standby unit of ship equipment has a certain failure rate. Additionally, cold standby system can be seen as a special case of warm standby system. After obtaining the reliability model for warm standby system, it is very convenient to construct a reliability model for cold standby system. On the other hand, most ship equipments are repairable. If any equipment in a standby system fails, ship crew or supporting personnel will repair the failed equipment in a timely manner to guarantee system availability, especially during a long voyage. Above all, the study on reliability model for warm standby repairable system is of great value to the extensive engineering application for ship equipment.

In the study on the warm standby system reliability with PH distribution, Gruruajan et al. ${ }^{[7]}$ explored two-component warm standby repairable system by assuming that the life of operating components and life of standby components were subject to PH distribution, there was only single equipment under repair, the life and service time of equipment under repair were subject to exponential distribution, and the repair time of repaired component was exponential distribution. Under these assumptions, the relevant reliability parameters of this system were obtained after analysis, e.g. reliability and availability. Pérez-Ocón et al. ${ }^{[8]}$ studied the warm standby system containing $\mathrm{n}$ units and having 1 operating unit. There was 1 repair platform, and it was assumed that the life and repair time of the operating unit were subject to PH distribution, and the life of standby unit was subject to exponential distribution, so that reliability parameters including steady-state availability and system operation duration were obtained. Wen et al ${ }^{[9]}$ took into account multiple vacations of repairmen in a system subject to the external impact of Poisson arrival process. Assuming that the life of operating components was subject to PH distribution, and the life of standby components featured exponential distribution, such indicators as probability of repairmen at work and failure rate were obtained. Barron et al. ${ }^{[10]}$ studied the $\mathrm{N}$-component warm standby system under the assumption that the life of components was subject to different PH distributions, the repair time of failed component was subject to geometrical distribution, and the repair cost of system was optimized on the basis of state.

As revealed in the above condition, most studies on warm standby system reliability assume that some random variables inside system are subject to $\mathrm{PH}$ distribution. As ship equipment is under complicated environment, and repair conditions are varying, the distribution of random variables 
including equipment life and repair time is diverse, which restricts the applicability of the above model. Hence, the study in this paper makes some improvements based on the existing studies. In a warm standby system with $\mathrm{n}$ units, 1 unit is operating and the other units are standby. Moreover, there is a repair platform, and the life of operating unit, life of standby units and repair time are subject to $\mathrm{PH}$ distribution, which further expands the applicability of the model.

\section{System Description and Assumptions}

Considering a repairable warm standby system consisting of $\mathrm{n}$ units, if 1 unit is available, the system is available. The system has 1 repair platform. The assumptions are as follows:

(1) The life of operating unit is subject to PH distribution, which is represented by $(\boldsymbol{\alpha}, \boldsymbol{T})$, and the order is $m$;

(2) The life of each standby unit is subject to PH distribution, which is represented by $(\boldsymbol{\beta}, \boldsymbol{S})$, and the order is $s$;

(3) The repair time of failed unit on the repair platform is subject to $\mathrm{PH}$ distribution, which is represented by $(\boldsymbol{\gamma}, \boldsymbol{L})$, and the order is $l$;

(4) The repair platform follows the strategy of first come first service, and can restore the failed unit to its original state after repair;

(5) The above random variables are independent from one another;

(6) Except the operating unit, the other units are in three possible states, i.e. standby, repair or wait for repair. A repaired unit is in the standby state. If there is not any other unit available after a unit is repaired, the unit will be immediately put into operation;

(7) The time needed to replace a failed unit with a standby unit is overlooked;

(8) The changeover switch is reliable.

\section{Model Analysis and Construction}

\section{Analysis on System States}

Based on the above system description and assumptions, the macrostate of system can be represented by the number of unavailable operating units. Let $K(t)$ be the number of unavailable units in a system at the time $t$, there is $K(t)=0,1,2 \mathrm{~L} n$.

Let $J(t)=\left\{j_{1}(t), j_{2}(t), \ldots, j_{k}(t), \ldots, i_{n-K(t)}(t)\right\}$ be the phase at which each available unit in the system locates at the time $t$; and let $I(t)$ be the phase at which the repair is happening. Hence, the change of system state represented by $\{K(t), J(t), I(t)\}$ forms a multidimensional Markov chain. The space of system state can be divided into three macrostates, i.e. $\Omega=H_{1} \cup H_{2} \cup H_{3}$. Macrostate $H_{1}$ means that all units in the system are available; macrostate $H_{3}$ implies that all units in the system are unavailable; macrostate $H_{2}$ is an intermediate state between $H_{1}$ and $H_{3}$. The three macrostates can be represented as follows:

$H_{1}=\left\{\left(0,\left(j_{1}(t), \mathrm{L} j_{p}(t), \mathrm{L} j_{n}(t)\right)\right)\right\}$, in which $j_{p}(t)$ stands for the phase the operating unit locates at, so there is $1 \leq j_{p}(t) \leq m$; and the phase of the other standby units is $1 \leq j_{y}(t) \leq s, y \neq p$.

$H_{2}=\left\{\left(k,\left(j_{1}(t), \mathrm{L} j_{p}(t), \mathrm{L} j_{n-k-1}(t)\right), i\right)\right\}$, which means that the system has $k$ unavailable units, and the repair is at phase $i$; each intact unit is at phase $j_{1}(t), \mathrm{L} j_{p}(t), \mathrm{L} j_{n-k-1}(t)$. The phase of the operating unit $j_{p}(t)$ satisfies $1 \leq j_{p}(t) \leq m$, while the phase of the other standby units is $1 \leq j_{y}(t) \leq s$, $y \neq p$.

$H_{3}=\{(n, i)\}$, which implies that $n$ units are unavailable, so the system is shut down; the repair is at phase $i, 1 \leq i \leq l$. 


\section{Analysis on System State Transfer Matrix}

After analyzing the system states, the transfer between system states is analyzed.

It is illustrated by the internal transfer of macrostates.

(1) $H_{1}$ internal transfer: When $k=0$, the system has no failed unit. The transfer involves the transfer of 1 operating unit and the transfer of $n-1$ standby units. The transfer matrix can be $64 \eta^{-1} 48$ represented by $\boldsymbol{T} \oplus \mathbf{S}^{\prime}$, in which $\mathbf{S}^{\circ}=\mathbf{S} \oplus \mathbf{S L} \oplus \mathbf{S}$.

(2) $\mathrm{H}_{2}$ internal transfer: When the system has $k$ units unavailable $(1 \leq k \leq n-1)$, either the phase of operating units or the phase of the repair changes at one time, and the phases should not change simultaneously, so their transfer is represented by $\boldsymbol{T} \oplus \boldsymbol{L}$. In the meantime, the phase of the other standby units $n-k-1$ may change. Thus, the transfer matrix of the whole system can be represented

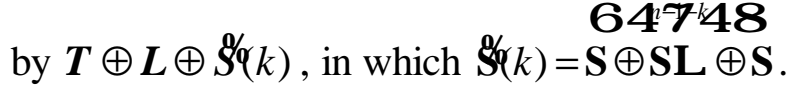

(3) $H_{3}$ internal transfer: At this time, the number of unavailable units in the system is $n$, so the system is shut down. At this time, there is only the transfer between the phases of the repair, which can be represented by $\boldsymbol{L}$.

Similarly, a matrix can be obtained for the transfer of macrostate $k$ to $k+1$ and $k-1$.

According to the above analysis, the state transfer matrix $\boldsymbol{Q}$ of the system is as follows:

$$
\boldsymbol{Q}=\left(\begin{array}{cccccc}
\boldsymbol{B}_{0,0} & \boldsymbol{B}_{0,1} & & & & \\
\boldsymbol{B}_{1,0} & \boldsymbol{A}_{1}^{(1)} & \boldsymbol{A}_{0}^{(1)} & & & \\
& \boldsymbol{A}_{2}^{(2)} & \boldsymbol{A}_{1}^{(2)} & \boldsymbol{A}_{0}^{(2)} & & \\
& & \mathrm{O} & \mathrm{O} & \mathrm{O} & \\
& & & \boldsymbol{A}_{2}^{(n-1)} & \boldsymbol{A}_{1}^{(n-1)} & \boldsymbol{B}_{n-1, n} \\
& & & & \boldsymbol{B}_{n, n-1} & \boldsymbol{B}_{n, n}
\end{array}\right)
$$

Each sub-matrix in $\boldsymbol{Q}$ is as follows:

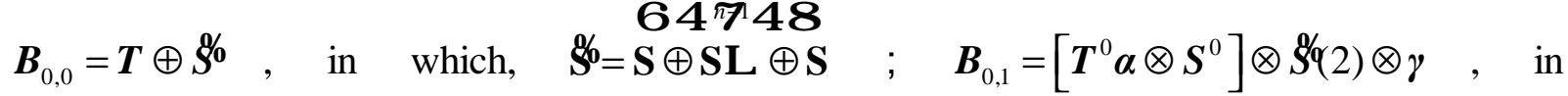

\section{$64 \pi^{\pi-2} 48$}

which, $\mathcal{S}(2)=\mathbf{S} \oplus \mathbf{S L} \oplus \mathbf{S} ; \boldsymbol{B}_{1,0}=\boldsymbol{I}(1) \otimes \boldsymbol{L}^{0} \otimes \boldsymbol{\beta}$, in which $\boldsymbol{I}(k)$ is the unit matrix with the number of dimensions $m \times s^{n-1-k} ; \boldsymbol{A}_{2}^{(k)}=\boldsymbol{I}(k) \otimes \boldsymbol{L}^{0} \boldsymbol{\beta} \otimes \boldsymbol{\gamma}$, in which, $2 \leq k \leq n-1 ; \boldsymbol{A}_{1}^{(k)}=\boldsymbol{T} \oplus \boldsymbol{S}(\mathrm{e} k) \oplus \boldsymbol{L}$, in which, $k=1,2, \mathrm{~L} n-1 ; \boldsymbol{A}_{0}^{(k)}=\left[\boldsymbol{T}^{0} \boldsymbol{\alpha} \otimes \boldsymbol{S}^{0}\right] \otimes S^{\prime}(k+1) \otimes \boldsymbol{I}$, in which, $k=1,2, \mathrm{~L} n-2 ; \boldsymbol{B}_{n-1, n}=\boldsymbol{T}^{0} \otimes \boldsymbol{I} ;$ $\boldsymbol{B}_{n, n-1}=\boldsymbol{L}^{0} \boldsymbol{\alpha} \otimes \boldsymbol{\gamma} ; \boldsymbol{B}_{n, n}=\boldsymbol{L}$ 。

\section{System Steady-state Probability Vector}

System steady-state probability vector is represented by $\pi=\left(\pi_{0}, \pi_{1}, \mathrm{~L}, \pi_{n-1}, \pi_{n}\right)$, which satisfies the following equations:

$$
\left\{\begin{array}{l}
\pi Q=0 \\
\pi e=1
\end{array}\right.
$$

The equations can be expanded into Equations (1) to (6).

$$
\begin{aligned}
& \pi_{0} \boldsymbol{B}_{0,0}+\pi_{1} \boldsymbol{B}_{0,1}=\mathbf{0} \\
& \pi_{0} \boldsymbol{B}_{0,1}+\pi_{1} A_{1}^{(1)}+\pi_{2} A_{2}^{(2)}=\mathbf{0} \\
& \pi_{k-1} A_{0}^{(k-1)}+\pi_{k} A_{1}^{(k)}+\pi_{k+1} A_{2}^{k+1}=\mathbf{0}, k=2,3, \mathrm{~L}, n-2 \\
& \pi_{n-2} A_{0}^{(n-2)}+\pi_{n-1} A_{1}^{(n-1)}+\pi_{n} B_{n, n-1}=\mathbf{0}
\end{aligned}
$$




$$
\begin{aligned}
& \boldsymbol{\pi}_{n-1} \boldsymbol{B}_{n-1, n}+\boldsymbol{\pi}_{n} \boldsymbol{B}_{n, n}=\mathbf{0} \\
& \boldsymbol{\pi}_{0} e+\boldsymbol{\pi}_{1} e+\mathrm{L}+\boldsymbol{\pi}_{n-1} e+\boldsymbol{\pi}_{n} e=1
\end{aligned}
$$

Equations (1), (2), (4) and (5) are all the boundary equations of the system.

After solving the above equations, the steady-state probability vector of system can be obtained.

\section{System Reliability Features}

\section{System Steady-state Availability}

After obtaining the steady-state probability vector of system, the steady-state availability of this warm standby system is the sum of the probabilities that the system is in each available microstate, that is:

$$
A=\sum_{k=0}^{n-1} \boldsymbol{\pi}_{k} \boldsymbol{e}=1-\boldsymbol{\pi}_{n} \boldsymbol{e}
$$

Meanwhile, we can obtain the unavailability of system, i.e. $\bar{A}=1-A=\boldsymbol{\pi}_{n} \boldsymbol{e}$.

\section{Mean Time between Failures of System}

Mean time between failures of system ${ }^{[12]}$ is an important indicator for evaluating the system reliability. In the relevant studies, the mean time between failures of standby system is mainly defined from two approaches:

Definition 1 It is the interval between the time when all units in a system are available (the system is in the macrostate 0) and the time when all units in the system are unavailable for the first time (the system is in the macrostate $n$ ).

Definition 2 It is the interval between the time when the repair of a unit is completed (the other units in the system are unavailable at this time) and the time when all units in the system are unavailable for the first time (the system is in the macrostate $n$ ).

Definition 1 mainly describes the average work time before the first failure of system, while Definition 2 focuses on the mean time between two system shutdowns due to failure after it operates for a long time. In this study, Definition 2 is employed.

According to Definition 2, when a system enters the steady state, the mean time between failures of the system is the interval between the time when the system changes from the macrostate $\mathrm{n}$ to the macrostate $\mathrm{n}-1$ and the time when it enters the macrostate $\mathrm{n}$ again. After $K(t)$ transfers from the macrostate $\mathrm{n}$ to the macrostate $\mathrm{n}-1$, the system starts operation. When $K(t)$ enters the macrostate $\mathrm{n}$ again, the system is shut down. Hence, the state space $H_{3}$ is the one-dimensional absorption state of system work time.

To calculate the distribution of time between system failures, a modified Markov process ${ }^{[13]}$ is employed on the basis of the Markov process established in Section 3.2. The process changes the original macrostate $\mathrm{n}$ into the absorption state, which is macrostate $n^{*}$, but the other macrostates remain unchanged. The mean time between failures of system is the time before entering the macrostate $n^{*}$.

Based on the above analysis and the definition of PH distribution, the mean time between failures of system is subject to $\mathrm{PH}$ distribution, and its infinitesimal generator $Q^{*}$ is as follows:

$$
\boldsymbol{Q}^{*}=\left(\begin{array}{c|ccccc|c} 
& 0 & 1 & & \mathrm{~L} & n-1 & n^{*} \\
\hline 0 & \boldsymbol{B}_{0,0} & \boldsymbol{B}_{0,1} & & & & 0 \\
1 & \boldsymbol{B}_{1,0} & \boldsymbol{A}_{1}^{(1)} & \boldsymbol{A}_{0}^{(1)} & & & 0 \\
& & \boldsymbol{A}_{2}^{(2)} & \boldsymbol{A}_{1}^{(2)} & \boldsymbol{A}_{0}^{(2)} & & 0 \\
\mathbf{M} & & & \mathrm{O} & \mathrm{O} & \mathrm{O} & 0 \\
n-1 & & & & \boldsymbol{A}_{2}^{(n-1)} & \boldsymbol{A}_{1}^{(n-1)} & \boldsymbol{B}_{n-1, n}^{*} \\
\hline n^{*} & 0 & 0 & 0 & 0 & 0 & 0
\end{array}\right)
$$


If the mean time between failures of system has non-reducible representation $(\boldsymbol{\delta}, \boldsymbol{U})$, there is $\boldsymbol{\delta}=(0, \ldots, 0, \boldsymbol{\alpha} \otimes \boldsymbol{\gamma})$, and:

$$
\boldsymbol{U}=\left(\begin{array}{ccccc}
\boldsymbol{B}_{0,0} & \boldsymbol{B}_{0,1} & & & \\
\boldsymbol{B}_{1,0} & \boldsymbol{A}_{1}^{(1)} & \boldsymbol{A}_{0}^{(1)} & & \\
& \boldsymbol{A}_{2}^{(2)} & 0 & 0 & \\
& & \mathrm{O} & \boldsymbol{A}_{1}^{(n-2)} & \boldsymbol{A}_{0}^{(n-2)} \\
& & & \boldsymbol{A}_{2}^{(n-1)} & \boldsymbol{A}_{1}^{(n-1)}
\end{array}\right)
$$

Obviously, $\boldsymbol{U}$ can be obtained by simply removing the $n+1$ line and the $n+1$ row in the original $\boldsymbol{Q}$ matrix.

On the basis of the above analysis, the mean time between failures of system is obtained as follows:

$$
M T B F=-\delta U^{-1} e
$$

\section{Failed Unit Arrival Rate of Repair Platform}

The unit arriving at the repair platform is the unit that fails in a system. It consists of two aspects, 1 . The operating unit fails, 2. The standby unit fails. Hence, the failed unit arrival rate of repair platform $v$ is the sum of the rates for the operating unit in a system and all the standby units in the system fail. After obtaining the steady-state probability of system, it is obtained that:

$$
v=\boldsymbol{\pi}_{0} \boldsymbol{F}^{0}+\sum_{k=1}^{n-k} \boldsymbol{\pi}_{k} \boldsymbol{F}^{0}(k)
$$

In which, $\boldsymbol{F}=\boldsymbol{B}_{0,0}, \boldsymbol{F}(k)=\boldsymbol{T} \oplus \stackrel{\&}{(}(k), \boldsymbol{F}^{0}=-\boldsymbol{F} \boldsymbol{e}$.

\section{Example Analysis}

This section utilizes an example to verify the correctness and applicability of model.

Considering a $n=3$ warm standby system, there is 1 operating unit and 1 repair platform.

Assuming that the life and repair time of the operating and standby units are subject to PH distribution, their non-reducible $\mathrm{PH}$ representation is as follows:

The life distribution of operating unit is $(\boldsymbol{\alpha}, \boldsymbol{T})$ :

$$
\boldsymbol{T}=\left(\begin{array}{ccc}
-1.750 & 0.170 & 0.013 \\
0.004 & -2.960 & 0.001 \\
0.093 & 2.266 & -3.646
\end{array}\right), \boldsymbol{T}^{0}=-\boldsymbol{T} \boldsymbol{e}=\left(\begin{array}{c}
1.567 \\
2.955 \\
1.287
\end{array}\right)
$$

The life distribution of standby units $(\boldsymbol{\beta}, \boldsymbol{S})$ :

$$
\begin{gathered}
\boldsymbol{\beta}=(0.460,0.540), \\
\boldsymbol{S}=\left(\begin{array}{cc}
-1.019 & 0.022 \\
0.015 & -1.015
\end{array}\right), \boldsymbol{S}^{0}=\left(\begin{array}{c}
0.997 \\
1.0
\end{array}\right) .
\end{gathered}
$$

The repair time distribution is $(\boldsymbol{\gamma}, \boldsymbol{L})$ :

$$
\boldsymbol{L}=\left(\begin{array}{ccc}
-4.863 & 0.019 & 0.126 \\
0.049 & -4.957 & 0.255 \\
0.019 & 0.007 & -4.832
\end{array}\right), \boldsymbol{L}^{0}=\left(\begin{array}{l}
4.718 \\
4.653 \\
4.806
\end{array}\right)
$$


Considering the number of unavailable units in the system, the state set of the system is represented by $\{0,1,2,3\}$. In the set, the state 3 is the state of system shutdown. The transfer between system states is as shown in Fig. 1:

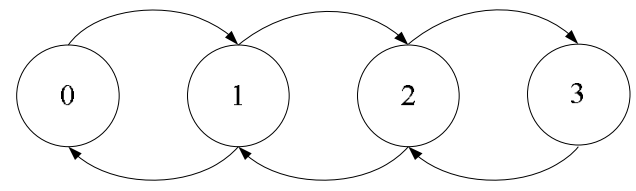

Fig. 1 Schematic diagram of system state transfer

The state transfer matrix $\boldsymbol{Q}$ of the system is as follows:

$$
\boldsymbol{Q}=\left(\begin{array}{cccc}
\boldsymbol{B}_{0,0} & \boldsymbol{B}_{0,1} & & \\
\boldsymbol{B}_{1,0} & \boldsymbol{A}_{1}^{(1)} & \boldsymbol{A}_{0}^{(1)} & \\
& \boldsymbol{A}_{2}^{(2)} & \boldsymbol{A}_{1}^{(2)} & \boldsymbol{B}_{2,3} \\
& & \boldsymbol{B}_{3,2} & \boldsymbol{B}_{3,3}
\end{array}\right)
$$

In which:

$\boldsymbol{B}_{0,0}=\boldsymbol{T} \oplus \mathcal{S}^{\prime}$, in which, $\mathcal{S}^{\mathbb{Q}} \boldsymbol{S} \oplus \boldsymbol{S} ; \boldsymbol{B}_{0,1}=\left[\boldsymbol{T}^{0} \boldsymbol{\alpha} \otimes \boldsymbol{S}^{0}\right] \otimes \mathcal{S}(2) \otimes \gamma$, in which: $\mathscr{S}(2)=\boldsymbol{S} ;$ $\boldsymbol{B}_{1,0}=\boldsymbol{I}(1) \otimes \boldsymbol{L}^{0} \otimes \boldsymbol{\beta}$, in which $\boldsymbol{I}(1)$ is a unit matrix with 6 dimensions ; $\boldsymbol{A}_{1}^{(1)}=\boldsymbol{T} \oplus \boldsymbol{S}(\mathrm{l}) \oplus \boldsymbol{L}$; $\boldsymbol{A}_{0}^{(1)}=\left[\boldsymbol{T}^{0} \boldsymbol{\alpha} \otimes \boldsymbol{S}^{0}\right] \otimes \boldsymbol{S}(2) \otimes \boldsymbol{I} ; \boldsymbol{A}_{2}^{(2)}=\boldsymbol{I}(2) \otimes \boldsymbol{L}^{0} \boldsymbol{\beta} \otimes \boldsymbol{\gamma}$, in which $\boldsymbol{I}(2)$ is a unit matrix with 3 dimensions ; $\boldsymbol{A}_{1}^{(2)}=\boldsymbol{T} \oplus \$(\rho) \oplus \boldsymbol{L} ; \boldsymbol{B}_{2,3}=\boldsymbol{T}^{0} \otimes \boldsymbol{I} ; \boldsymbol{B}_{3,2}=\boldsymbol{L}^{0} \boldsymbol{\alpha} \otimes \boldsymbol{\gamma} ; \boldsymbol{B}_{3,3}=\boldsymbol{L} 。$

The above equations are substituted into Equation (1)-Equation (6), we can obtain the equations for steady-state probability vector:

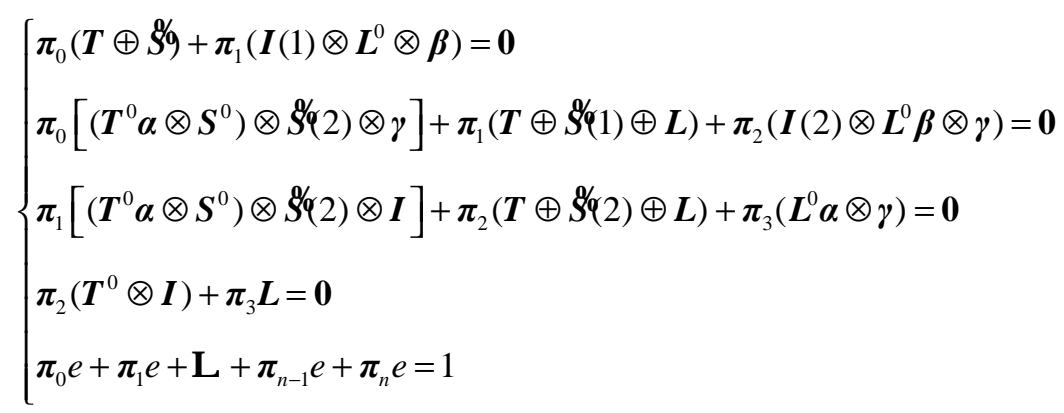

Following the relevant steps of matrix analysis method in Section 3.3, the steady-state probability vector of system can be solved.

With Equation (17), it is obtained that the system steady-state availability is $A=0.9934$.

The mean time between failures of system has the non-reducible $\mathrm{PH}$ representation $(\boldsymbol{\delta}, \boldsymbol{U})$, in which:

$$
\boldsymbol{\delta}=(0, \ldots, 0, \boldsymbol{\alpha} \otimes \boldsymbol{\gamma}), \boldsymbol{U}=\left(\begin{array}{ccc}
\boldsymbol{B}_{0,0} & \boldsymbol{B}_{0,1} & \\
\boldsymbol{B}_{1,0} & \boldsymbol{A}_{1}^{(1)} & \boldsymbol{A}_{0}^{(1)} \\
& \boldsymbol{A}_{2}^{(2)} & \boldsymbol{A}_{1}^{(2)}
\end{array}\right)
$$

Based on Equation (18), we can calculate and obtain $M T B F=1.2157$. Based on Equation (19), it is calculated to obtain $v=0.0472$. 
As revealed in the reliability indicators in this example, all reliability indicators satisfy the requirements for system reliability, so it means that the reliability model for $\mathrm{N}$-component warm standby repairable system with $\mathrm{PH}$ distribution can accommodate all kinds of random distributions, so its applicability is further expanded.

\section{Acknowledgements}

This work was financially supported by National Nature Science Foundation under Grant 71501183.

\section{References}

[1] Ayse Sena E, Tarkan T, Geert-Janvan H. Optimizing usage and maintenance decisions for k-out-of-n systems of moving assets[J]. Naval Research Logistics, 2017, 64(5): 418-434.

[2] Gregory L, Liudong X, Yuanshun D. Optimization of Component Allocation/Distribution and Sequencing in Warm Standby Series-Parallel Systems[J]. IEEE Transactions on Reliability, 2017, 66(4): 980-988.

[3] Yadavalli V S S, Vaidyanathan V S, Chandrasekhar P, Abbas S. Applications of quadrivariate exponential distribution to a three-unit warm standby system with dependent structure[J]. Communications in Statistics - Theory and Methods, 2017, 46(14): 6782-6790.

[4]Serkan E. The effectiveness of adding cold standby redundancy to a coherent system at system and component levels[J]. Reliability Engineering and System Safety, 2017, 165(4): 161-165.

[5] Mohamed Salah E. Stochastic Behavior of a Two-Unit Cold Standby Redundant System Under Poisson Shocks[J]. Arabian Journal for Science and Engineering, 2017, 42(7): 3043-3053.

[6] Yongjin Z, Yanjun Z. Reliability analysis of a parallel dependent system with a single cold standby unit[J]. International Journal of Industrial and Systems Engineering, 2016, 23(2): 166-180.

[7] Srinivasan B, Gruruajan M. A Complex Two-unit System with Random Breakdown of Repair Facility[J]. Microelectronics Reliabilty, 1995, 35(2): 299-302.

[8] Montoro-Cazorla D, Pérez-Ocón F. A Multiple Warm Standby System with Operational and Repair Times Following Phase-type Distributions[J]. European Journal of Operational Research, 2006, 169: 178-188.

[9] Yanqing W, Lirong C, Shubin S, Baoliang L. A multiple warm standby $\delta$-shock system with a repairman having multiple vacations[J]. Communications in Statistics - Simulation and Computation, 2017, 46(4): 3172-3186.

[10] Barron Y, Yechiali U. Generalized control-limit preventive repair policies for deteriorating cold and warm standby Markovian systems[J]. IISE Transactions, 2017, 49(11): 1031-1049.

[11] Baum D, Breuer L. An Introduction to Queueing Theory and Matrix- Analytic Methods[J]. OR Spektrum, 1983, (5): 25-32.

[12] Jing-hua C, Kan C. An introduction to mathematical of reliability[M], Beijing: Higher Education Press, 2006.

[13] QI Ming H. Fundamentals of matrix-analytic methods[M]. London: Springer, 2013. 